\title{
The temporal distribution of stimulus information in the visual evoked potential
}

\author{
ROBERT F. STANNERS and STEPHEN CARVER \\ Oklahoma State University, Stillwater, Oklahoma
}

\begin{abstract}
Research in psychophysics (Bloch's law) and perceptual experiments concerned with the integration of successively presented stimuli suggest that the perception of form is a process that occurs over a period of as much as 200-300 msec. Such results prompted the question of whether the visual evoked potential (VEP) might contain information about the distribution over time of perceptual processing. Subjects viewed lines formed from combinations of three lengths and four angles while the EEG was recorded. Analysis of the VEPs indicated that the length and angle of the lines produced temporal distributions of information in the VEP and that the distributions for length and angle were somewhat different. The major difference was that the processing of angle begins earlier and is completed sooner than the processing of length. A conclusion of the experiment was that an alternative or supplement to analyzing VEPs for specific waveform features is to consider the encoding of stimulus information in the VEP as a density or concentration over time.
\end{abstract}

Our principal objective in the present experiment was to investigate the possibility that information from a visual stimulus may be encoded in the visual evoked potential (VEP) in such a way that the information about the stimulus is distributed over a fairly substantial time interval, possibly several hundred milliseconds. Another objective was to investigate whether different physical features of the stimulus-in the present case length and angle of a line-might have different temporal distributions in the VEP.

Bloch's law, a well-established finding from visual psychophysics, suggests some approximate intervals for the temporal integration of information in the visual system. Bloch's law states that for some visual tasks, a reciprocity relationship exists between the intensity and duration of a visual stimulus. For example, within a certain time interval, a tradeoff between duration and intensity will yield essentially the same results for perceived brightness. Kahneman and Norman (1964) found the critical (reciprocity) interval for perceived brightness to be approximately $100 \mathrm{msec}$, which is within the range of findings from numerous other experiments. In the same study, the critical interval for a form perception task (identification of zeros and ones) was also investigated. The critical interval for the form perception task, approxi-

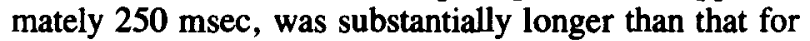
perceived brightness. In another experiment (Kahneman, Norman, \& Kubovy, 1967), the identification task was used in conjunction with dichoptic stimulation. Here the critical interval was at least $300 \mathrm{msec}$.

The authors decided that equal contributions were made to the experiment, so order of authorship was decided by a coinflip. Send for reprints to: Robert F. Stanners, Department of Psychology, Oklahoma State University, Stillwater, OK 74078.
Another line of research that demonstrates temporal integration of form information in the visual system is exemplified in some experiments by Eriksen and Collins (1967, 1968). Subjects were shown brief presentations of two-dot patterns. When presented separately, the dot patterns appeared to be random; when presented simultaneously, however, they formed an easily recognizable trigram. Successive presentations of the dot patterns with a varying interstimulus interval indicated that identification of the trigram was possible with interstimulus intervals of up to $300 \mathrm{msec}$.

Although previous VEP research has not considered the temporal integration issue, there is evidence that some information about the physical characteristics of a visual stimulus is encoded in the VEP. John, Herrington, and Sutton (1967) found that variations in the shape of a stimulus (a four-sided figure oriented as a square or diamond) produced different positive components in the VEP. Other results in the same study showed that size variations of the same-shaped stimulus produced amplitude and latency differences. John (1976) varied the size of letters of the alphabet and found amplitude differences in the VEP at approximately $100 \mathrm{msec}$.

Several more recent experiments have resulted in VEP components associated with selective attention to individual attributes of a multiattribute stimulus. For example, Previc and Harter (1982) found several time points in the VEP that showed amplitude differences linked to selective attention directed toward the spatial frequency and orientation of a visual stimulus. Hillyard and Münte (1984) found a number of amplitude differences associated with selective attention to location and color. In the present experiment, we are not directly concerned with the theoretical issues addressed in the selective attention experiments, but rather with the implication that the VEP does 
contain some specific information about the individual attributes of a multiattribute stimulus.

Research on Bloch's law and the integration of successively presented stimuli suggest that it might be profitable to consider rather long time intervals of the VEP, in order to gain information about the characteristics of a visual stimulus. In the present study, a method of data analysis was developed and an experiment conducted to investigate the question of the temporal distribution of stimulus information in the VEP.

\section{METHOD}

\section{Subjects}

Sixteen graduate and undergraduate students served as subjects. All subjects either had normal vision or wore corrective lenses.

\section{Stimulus Materials}

The stimuli consisted of the set of 12 lines formed from the combinations of three lengths and four angles. Each line was short $(3 \mathrm{~cm})$, medium $(7 \mathrm{~cm})$, or long $(12 \mathrm{~cm})$. The lines were presented on a video monitor at a distance of $1.1 \mathrm{~m}$ subtending visual angles of $1.54^{\circ}, 3.59^{\circ}$, and $6.15^{\circ}$ for small, medium, and long, respectively. Each line was presented at an orientation of $0^{\circ}$ (horizontal), $30^{\circ}, 60^{\circ}$, or $90^{\circ}$ (vertical). The lines were centered on the monitor at a brightness level of $151 \mathrm{~cd} / \mathrm{m}^{2}$. The stimuli were generated by an Apple II computer in its high-resolution graphics mode and displayed on a 12-in. high-resolution monitor with a P31 phosphor screen.

\section{Procedure}

The subjects were seated in a darkened and electrically shielded room. They were instructed to fixate an illuminated point at the center of the video monitor and to refrain from blinking or moving while the stimulus was on the screen. The subjects were dark-adapted for $8 \mathrm{~min}$ and then given a series of 12 familiarization trials ( 1 for each line). On each familiarization trial, a line was presented for $1 \mathrm{sec}$ in place of the fixation point, and a descriptive name was presented below it (e.g., "short, 30 degrees," "long, 60 degrees").

After the familiarization trials, the subjects were given 12 practice trials ( 1 for each stimulus line) and 192 data trials ( 16 for each stimulus line). The practice and data trials were differentiated only by the fact that EEG data were not collected on the practice trials.

A trial began with the presentation of a .5-sec, 1000- $\mathrm{Hz}$ tone. The tone signaled the subject that a trial could be started with the actuation of a hand-held button switch. The switch initiated an interval that varied randomly between .5 and $1 \mathrm{sec}$. If the scalp potential exceeded a $32-\mu \mathrm{V}$ threshold, used to detect muscle artifact, another interval was initiated. If the threshold voltage was not exceeded during the interval, then the stimulus line was presented for $1 \mathrm{sec}$. A potential that exceeded the threshold during presentation of the line resulted in the EEG data being discarded and the stimulus being re-presented in a randomly determined position in the subsequent trials.

Data collection began with the presentation of the stimulus line and continued for $400 \mathrm{msec}{ }^{1}$ The subjects were instructed to name the stimulus line after it left the screen. The names were the descriptions used in the familiarization trials, a length descriptor followed by an angle descriptor (e.g., "long, ninety"). The naming task was used to ensure attention to the stimulus, and it proved to be quite easy, with performance near $100 \%$ for all subjects. The intertrial interval was 5-7 sec, during which time the subject was allowed to readjust his position. The total time for all trials was approximately $50 \mathrm{~min}$.

The stimulus lines were presented in an order randomly determined for each subject, with the following constraints: The same stimulus was not presented twice in succession, and the distribution of each line was approximately uniform over the complete series.

A single monopolar electrode attachment was made at the posterior pole of the right occipital lobe, $2 \mathrm{~cm}$ above and $2 \mathrm{~cm}$ to the right of the inion (02 in the International [10-20] scheme). The right earlobe (A2) was used as the reference and the left earlobe (A1) as the ground.

EEG signals were amplified with a Grass Instruments Model 7D chassis fitted with wideband $\mathrm{AC}$ preamplifier and integrator unit (Model 7P3B), which drives a DC amplifier (Model 7DAF). The low-pass filter setting was $35 \mathrm{~Hz}$ (half amplitude down), and the high-pass filter had a time constant of $.3 \mathrm{sec}$. The EEG was sampled at the rate of $1,000 / \mathrm{sec}$ and digitized by a Burr-Brown data acquisition system (Model SDM 853), which resulted in a resolution of $.5 \mu \mathrm{V}$. The digitized data were stored on the disk of an Apple II computer, which was also used to present the stimuli and control the timing of the experiment.

Each of the 192 records was visually inspected for all subjects. None of the records appeared to contain substantial artifacts, and all were used in the averaging process. The averaging was done over the 16 records for each of the 12 stimulus lines.

\section{RESULTS}

\section{Data Analysis}

The starting data base was a set of 12 VEPs (one for each stimulus line) for each of the 16 subjects. Each VEP consisted of 400 data points, one value per millisecond.

The overall objective of the data analysis was to ascertain whether length and angle information from the stimulus lines had a temporal distribution in the VEPs. The basic assumption of the analysis was that length and angle information is encoded in the VEP in such a way that the similarity (or difference) relations among the stimulus lines are reflected in the similarity (or difference) relations among the VEPs. For example, on this assumption, the VEP for the short $0^{\circ}$ line should be more similar to the VEP for the medium $0^{\circ}$ line than it would be to the VEP for the long $0^{\circ}$ line. If such relationships held in general, then a complete matrix of pairwise comparisons between VEPs should contain the length and angle information. The problem for the analysis then becomes one of constructing the appropriate similarity matrices and extracting the length and angle information.

The data analysis proceeded in three steps. The first step was to intercorrelate the 12 VEPs for each subject over several time intervals. Each intercorrelation matrix may be viewed as reflecting the pattern of similarities among all pairwise comparisons of the VEPs for the 12 stimulus lines. Differences among the matrices reflect the different time intervals over which they were computed. The first interval covered the period from 1 to $50 \mathrm{msec}$. The second ran from 1 to 100 ; each succeeding matrix was produced by extending the interval by $50 \mathrm{msec}$ until the 400 -msec point was reached. ${ }^{2}$

The addition of VEP data by progressively moving the ending point of the interval provides one view of the temporal distribution of length and angle information. Another view was provided by the systematic deletion of data from the beginning of the VEP. This series of intercorrelation 
matrices was produced by moving the starting point to $51 \mathrm{msec}$ and then extending the interval by $50-\mathrm{msec}$ increments. The next series was produced by moving the starting point to $101 \mathrm{msec}$ and again taking $50-\mathrm{msec}$ increments. Proceeding in this fashion, the last intercorrelation matrix was for the interval 351-400, which resulted in a total of 36 matrices for each subject.

The second step in the analysis was to represent the pattern of pairwise similarities represented by the correlations in a simpler, more comprehensible manner. This was accomplished by applying three-way multidimensional scaling (MDS) to each of the 36 sets of intercorrelation matrices. MDS represents the structure of similarities in a correlation matrix as a set of points (one point for each stimulus line in the present case) in multidimensional space-in effect, a map. Two possibilities were of particular interest in the present study. One was that the dimensions of length and angle of the lines might be represented in the dimensions of the MDS maps. Another possibility was that the different time intervals over which the analyses were conducted might produce MDS maps that provided information on the time course of stimulus processing.

The MDS procedure was an algorithm called COSPA (Schönemann, James, \& Carter, 1979), which has the advantage of allowing statistical tests of two assumptions (the common space assumption and the diagonality assumption) that are important in the interpretation of scaling results. The test for common space assesses the degree of agreement between the scaling results for individual subjects and the overall result for the group. In the present study, the common space test was upheld at or beyond the .05 level for all but one of the 36 analyses; the exception was the interval 101-200 msec. Thus, in every case but one, the matrices for the individual subjects showed a beyond-chance level of commonality.

The diagonality test evaluates whether the data fit a model in which the dimensions can be regarded as orthogonal. The diagonality assumption was not upheld in any of the MDS analyses. If length and angle information are present in the MDS dimensions, such information cannot be considered orthogonal. The dimensions of the MDS maps were derived with principal components analysis. Therefore, each succeeding dimension represents an independent and decreasing proportion of the total variance in the VEPs.

The MDS maps, in the present case in three dimensions, represented a substantial simplification of the information in the intercorrelation matrices. Still, 36 three-dimensional MDS maps cannot very easily be evaluated by visual inspection. This state of affairs led to the third and final step in the data analysis, a procedure for quantitatively assessing the "goodness" of the maps.

This procedure goes directly back to the basic assumption of the data analysis: the ordinal similarity relations among the stimulus lines should result in a corresponding set of ordinal relations among the VEPs. If this assumption was correct, then a predictable ordering of points should occur in the MDS maps. For example, for length, the ordering of points small, medium, large (or the reverse) would be predicted in the MDS maps for each angle. Likewise, for angle, the ordering $0^{\circ}, 30^{\circ}, 60^{\circ}$, $90^{\circ}$ (or the reverse) would be predicted for each line length. On the basis of these expectations, an error score was calculated for length and angle for each dimension of each MDS map. To take a specific case with small, medium, large as "correct," an ordering of points medium, small, large would constitute a single error for length (only "medium-small" is incorrect). The ordering large, small, medium would constitute two errors (both "large-small" and "large-medium" are incorrect). Summing the ordering errors for size over the four angles, a total error score was calculated for each dimension of each map. An analogous procedure was followed to generate error scores for angle.

The error scores provided a quantitative description of the ordering aspect of the maps, though thus far without a baseline against which to evaluate whether a given score was unusually low. To address this problem, we calculated the exact probability (on a chance basis) of each value of an error score. Consider the case of zero errors for length. There are six possible orderings of the points small, medium, large for each of the four angles. Thus, $\operatorname{Pr}\left(\right.$ zero errors) $=2(1 / 6)^{4}=.0015$. The fact that there is no a priori basis for deciding whether the ordering small, medium, large or the reverse is correct was dealt with by the inclusion of the factor 2 in the equation. In effect, the symmetric values in the probability distribution were added together (a two-tailed test rather than a unidirectional test). The counterpart of this example for angle is $\operatorname{Pr}$ (zero errors) $=2(1 / 24)^{3}=.0001$. In similar fashion, the exact chance probabilities for the other error scores were calculated. The exact probabilities for length and angle are presented in Table 1, along with the cumulative values that provide the probabilities of a given error score plus all lower scores. ${ }^{3}$

Table 1

Exact and Cumulative Probabilities for Error Scores and Percentages

\begin{tabular}{|c|c|c|c|c|c|c|c|}
\hline \multicolumn{2}{|c|}{$\begin{array}{l}\text { Error } \\
\text { Score }\end{array}$} & \multicolumn{2}{|c|}{$\begin{array}{c}\text { Error } \\
\text { Percentage* }\end{array}$} & \multicolumn{2}{|c|}{$\begin{array}{c}\text { Exact } \\
\text { Probability }\end{array}$} & \multicolumn{2}{|c|}{$\begin{array}{l}\text { Cumulative } \\
\text { Probability }\end{array}$} \\
\hline ingle & Length & Angle & Length & Angle $\dagger$ & Length $\ddagger$ & Angle & Length \\
\hline 0 & 0 & 0 & 0 & .0001 & .0015 & .0001 & .0015 \\
\hline 1 & 1 & 11 & 17 & .00 & .01 & .0014 & .0138 \\
\hline 2 & 2 & 22 & 33 & .0061 & .049 & .0075 & .0632 \\
\hline 3 & 3 & 33 & 50 & .0195 & .1296 & .0270 & .1928 \\
\hline 4 & 4 & 44 & 67 & .04 & .2469 & .0752 & .4397 \\
\hline 5 & 5 & 55 & 83 & .09 & .3580 & .1715 & .7977 \\
\hline 6 & 6 & 67 & 100 & .1613 & .2022 & .3328 & .9999 \\
\hline 7 & & 78 & & .2305 & & .5633 & \\
\hline 8 & & 89 & & .2843 & & .8476 & \\
\hline 9 & & 100 & & .1523 & & .9999 & \\
\hline
\end{tabular}

*Error percentages rather than scores were used in Figure 1, so that angle and length could be placed on the same scale. TThe probąbilities for error scores of 0-8 for angle are the sums of the symmetric values in the probability distribution. There is no symmetric value for the error score of 9 . $¥$ The probabilities for error scores of 0-5 for length are the sums of the symmetric values in the probability distribution. There is no symmetric value for the error score of 6 . 

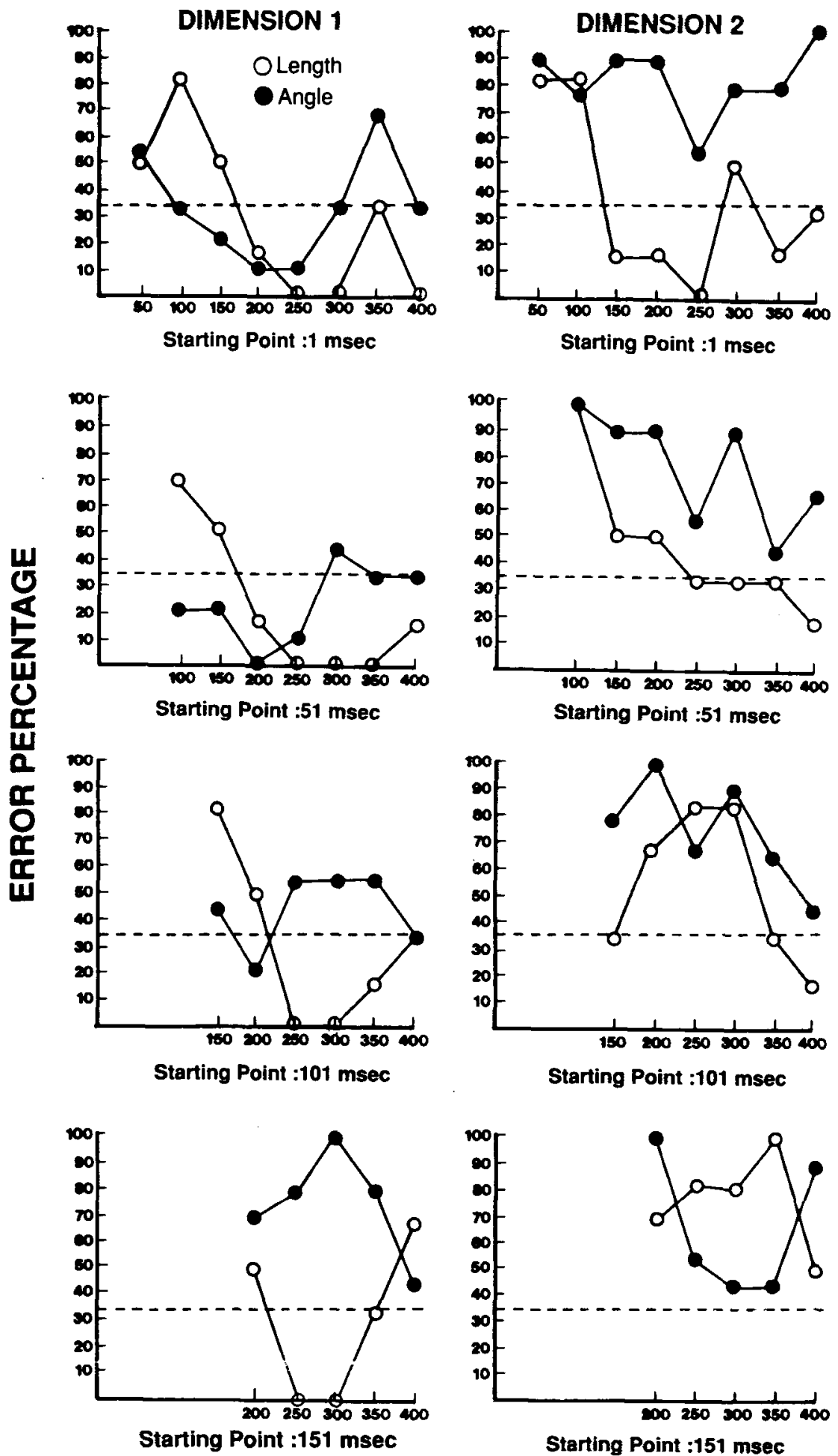

ENDING POINT

Figure 1. Error percentage as a function of ending point and starting point. 


\section{Plots of Error Scores}

The results of the error analysis are presented in Figure 1. Each data point is the percentage of ordering errors along a dimension of an MDS map defined by a particular time interval over which the intercorrelation matrix was calculated. Within panels, VEP data are being added, and across panels, data are being deleted from the beginning point of the VEPs. The dotted line at 33\% defines a point at and below which error scores are improbable by an approximate .05 (cumulative probability) standard. The actual values are .0632 for length and .0270 for angle. Exact and cumulative values for any error percentage may be read from Table 1 .

On the first dimension of the MDS maps, (left column of Figure 1), the first time interval to show a significantly low error score for angle was the one from 1-100 msec. Extending the ending point of the interval beyond 100 produced significantly low error scores out to the 300 msec point. The interval $1-350$ produced a relatively highs error percentage, but the score dropped again at 1-400. With the starting point set at 51 , all longer intervals produced low error scores for angle except for the 51-300 interval. Starting at 101 produced two significant intervals, 101-200 and 101-400. Moving the starting point out to 151 or beyond produced no significantly low error scores for angle.

For length on the first dimension, error scores do not become significantly low until the interval 1-200 and then remain low for later ending points. With a starting point of 51 , the error percentage becomes significantly low at 51-200 and remains low for later ending points. Deleting the data up to $101 \mathrm{msec}$ requires an ending point of 250 or beyond to generate low error scores. Unlike angle, length still produces significantly low error scores with a starting point as late as 151 , but this is the latest starting point to produce any significant values.

The plots for the second dimension of the MDS maps (right column of Figure 1) produced a very straightforward result for angle. None of the time intervals produced a significantly low error score. Length information, however, is represented on the second dimension. With a starting point of $1 \mathrm{msec}$, all intervals having an ending point of at least 150 produced a significantly low error score, with the exception of the interval ending at $300 \mathrm{msec}$. Deleting VEP data up to $51 \mathrm{msec}$ requires an ending point of 250 or later. The error percentage function for length assumed a rather different shape when the starting point was moved to 101 . Here an ending point of 150 produced a low error score as well as ending points of 350 and 400 . With all data prior to 151 deleted, length generated no low error scores. However, when the starting point was moved to 201 (not plotted), significantly low error scores (33\%) were found for ending points of 350 and 400 . The last starting point for which any significantly low error scores were found was 251 (not plotted); ending points of 300 and 400 both produced $33 \%$ errors.
The third dimension of the MDS maps yielded no significantly low error scores for angle or length for any combination of starting and ending points.

\section{DISCUSSION}

The common space tests, significant in 35 out of 36 cases, provide assurance that the overall MDS maps are not simply amalgams of unrelated correlation matrices for individual subjects. The common space tests reflect the consistency among subjects in the patterning of similarities among the VEPs for the various stimulus lines.

The diagonality test, which was not upheld in any of the 36 scaling analyses, indicates that length and angle information are not orthogonally encoded in the VEP, at least in the kind of information extracted by the intercorrelations and MDS. The lack of orthogonality is apparent in Figure 1, in that both length and angle information appear on the first dimension. The portion of length information uncorrelated with that for angle appears on the second dimension.

Two general features of the results are as follows: (1) a single dimension of the MDS analyses was sufficient to capture all the angle information insofar as the error scores are concerned, and (2) two dimensions captured both length and angle information. Error scores changed abruptly after one dimension for angle and two dimensions for length, from several scores being significant to no significant scores. These results accord with what would be expected from a procedure involving principal components analysis, which extracts maximum, independent variance components. If variations in the VEP data beyond the general noise level are produced by the experimental manipulations, then one would expect to see the results of the manipulations in the earliest components.

The stimulus information does have a fairly wide temporal distribution in the VEPs. For angle, only one interval as short as $\mathbf{5 0} \mathrm{msec}$ produced a significantly low error score $(51-100)$. Two such intervals occurred for length, both on the second dimension (101-150 and 250-300). All but one of the intervals showing perfect ordering were $150 \mathrm{msec}$ or more in length.

The three error functions for angle that contain significantly low error scores share in common the characteristics that they decrease, increase, and then decrease again. The greater the amount of the angle information in the VEPs, the more it would affect the pattern of intercorrelations among them, which in turn would be reflected in the MDS maps as a decrease in error scores. The initial decrease in the error functions followed by an increase can be interpreted as an indication of where in the interval angle information is most concentrated. An initial lengthening of the analysis interval had the effect of increasing the amount of angle information in the VEP data. A declining concentration of angle information in the intermediate portion of the interval is indicated by the fact that lengthening the analysis interval beyond $250 \mathrm{msec}$ 
produced an increase in error scores. Perceptual processing of the angle information appears to be largely complete by about $250 \mathrm{msec}$.

A final decrease in error scores for angle to a significant level occurs for all of the first three starting points, suggesting that the effect may not be simply a statistical fluke. The final decrease, coming as it does fairly late, could indicate a change from perceptual processing to a recognition or decision process that is linked to earlier perceptual analysis. In any event, the inclusion of the later portion of the VEPs has the effect of "improving" the pattern of correlations among the VEPs such that the correlations better represent the ordering relations among the angles.

There is also an effect of deleting data from the beginning of the VEP. The general trend is an increase in error scores (across panels of the graph), which would indicate that early VEP data ( $100 \mathrm{msec}$ or earlier) contains a relatively high concentration of angle information.

The temporal distribution of length information on the first dimension is shifted somewhat to the right relative to that for angle. One indication is that later ending points are required to produce significantly low error scores. It is also true that deleting early data produces a substantially smaller increase in error scores for length than for angle, and that more data can be deleted (up to $150 \mathrm{msec}$ ) and the remaining intervals still have some significantly low error scores.

As was the case for angle, there is an initial decrease in error scores with a lengthening of the analysis interval. Following the interpretation for angle, this decrease would reflect a greater amount of length information. Continuing to extend the length of the analysis interval results in an increase in error scores (more pronounced at the later starting points). In line with the interpretation for angle, this would indicate a lower concentration of length information as perceptual processing reaches completion. The final decrease in error scores observed for angle does not occur for length.

Deleting data from the beginning of the VEPs eventually has the effect of raising error scores for length to a nonsignificant level, but more data (up to $200 \mathrm{msec}$ ) must be deleted than was the case for angle. Length information is concentrated in the first half of the analysis interval.

On the second dimension, for the first two starting points, length shows functions similar to those on the first dimension. Error scores generally decrease with a lengthening of the analysis interval, indicating a greater amount of length information. Deleting early data (up to $51 \mathrm{msec}$ ) generally increases error scores presumably by a reduction in information from early processing.

The error function with the starting point of 101 is problematic because of the significant error score for 101-150 coupled with a nonsignificant score for 51-150 (previous panel). Deleting early data (1-50) seems to have the effect of reducing the amount of angle information (increasing error scores), yet the deletion of data that occurs between 51 and 100 has an opposite effect. If the score for 101-150 is taken as a fluke, then, beyond the first two starting points, only the longest intervals (ending on 400 ) produce significantly low error scores on a fairly consistent basis. Intervals ending on 400 produce significantly low error scores with five of the eight starting points. ${ }^{4}$ Overall, the results for the second dimension seem to indicate that early data and late data have a relatively high amount of length information. Perhaps, as was suggested for angle on the first dimension, a shift from one type of processing (perceptual analysis) to another (recognition or decision) is indicated.

There was an attempt to relate the results of the MDS and error analyses to amplitude and latency differences among the VEPs for the various stimulus lines. The possibility of interest was that intervals associated with very low error scores might show visually apparent changes in the waveforms associated with length and angle.

Subjects were chosen whose individual scaling results were similar to the group results for selected time intervals. The selected intervals were those that showed significantly low error scores and were also relatively short. Differences in amplitude would seem highly unlikely to persist for intervals beyond $100 \mathrm{msec}$. Studies in which amplitude and latency were investigated for their linkage to the shape and size of the stimulus employed (John, 1976; John et al., 1967) contained much shorter analysis intervals. The basis for choosing subjects was the value of their $v$ statistics for the selected intervals $(51-100$ for angle and 151-250 for length on the first dimension). The $v$ statistics are used in the test of common space; they measure the proportion of variance in the individual's scaling results that can be accounted for by the group results. Three subjects were selected who had $v$ statistics ranging from .66-.87 for the appropriate intervals. The general result of comparing VEPs for the stimulus lines was that no indication could be found of any consistent relationship between amplitude and latency differences on the one hand and size and angle on the other. That is, the ordering of latencies and amplitudes over the selected intervals failed to show correspondence with the ordering of angles and lengths.

In retrospect, there seem to be at least two reasons why the results of the MDS and error analysis would not necessarily indicate amplitude or latency differences. First, MDS is sensitive to consistency in a complex pattern of pairwise comparisons. In the present case, the analysis reflects the consistency across subjects of the patterning of correlation coefficients representing pairwise comparisons among the VEPs for the 12 stimulus lines. The scaling picks up patterns in relatively long-term trends in the way that the VEPs track one another. This is not the kind of information that translates in any direct way into amplitude or latency differences. Another aspect of the results is that $94 \%$ of significantly low error scores occurred for intervals $100 \mathrm{msec}$ or more in length. All but one of the perfect orderings were for intervals of $150 \mathrm{msec}$ or more. Amplitude differences do not generally persist for such long periods. The results of the scaling and error anal- 
yses make it clear that there were systematic differences among the VEPs for the various lines, but the nature of these differences was complex and was distributed over substantial time intervals.

\section{CONCLUSIONS}

Evidence from experiments in psychophysics and perception indicates that the visual processing of form is an operation extended over time. The results of the present experiment tend to confirm this view, in that they show temporal distributions of stimulus information in the VEP; the general form of these distributions consists of an increase of information early in the analysis interval and then a gradual decrease of information after 200 300 msec. Different features of the stimulus were shown to have different temporal distributions of information in the VEP; the major difference was that the processing for angle began sooner and ended sooner than that for length. The present experiment indicates that there is sufficient information in the VEP to differentiate among three levels of length and four levels of angle, but that to do so, it is necessary to consider substantial time segments of the VEP. Overall, the current results suggest a view of the encoding of information in the evoked potential as a concentration or density over time rather than a specific feature of the waveform within a narrow time band. Such a view would seem to expand the possibilities for extracting information from evoked potential data.

The present method could be adapted to investigate more specific features of the waveform over varying time intervals. Instead of using the correlation coefficient, which is an overall measure of waveform similarity, a similarity index could be based on a particular feature of the waveform. For example, a similarity index could be based on the average amplitude difference between waves over a given analysis interval, and a separate index could be calculated for each of several intervals. Average amplitude differences that were spread out over fairly long time intervals might very well not be visually apparent, because the waves might have crossed and recrossed. MDS analysis of the similarity matrices could extract information about consistent (across subjects) amplitude differences and depict it in a somewhat simplified form, as a set of MDS maps. The present error analysis could be used if there were some basis for predicting a particular ordering of amplitudes among the evoked potentials. This approach could be extended to other waveform features through the construction of appropriate similarity indices.
The pairwise comparison method might also be adapted to focus on portions of the waveform substantially smaller than those considered in the present experiment. For example, if theoretical considerations dictated that a particular feature occur within, say, a 100 -msec interval, the analysis interval might be made only $25 \mathrm{msec}$ long, with an increment of $10 \mathrm{msec}$. This approach could provide a relatively fine-grained look at a small portion of the evoked potential.

\section{REFERENCES}

Bradley, J. V. (1968). Distribution-free statistical tests. Englewood Cliffs, NJ: Prentice-Hall.

ERIKSEN, C. W., \& Collins, J. F. (1967). Some temporal characteristics of visual pattern perception. Journal of Experimental Psychology, 74, 476-484.

ERIKSEN, C. W., \& Collins, J. F. (1968). Sensory traces versus the psychological moment in the temporal organization of form. Joumal of Experimental Psychology, 77, 376-382.

Hilly ARd, S. A., MüNTE, T. F. (1984). Selective attention to color and location: An analysist with event-related brain potentials. Perception \& Psychophysics, 36, 185-198.

JoHN, E. R. (1976). A model of consciousness. In G. E. Schwartz \& D. Shapiro (Eds.), Consciousness and self-regulation (pp. 1-50). New York: Plenum.

John, E. R., Herrington, E. R., \& Sutton, S. (1967). Effects of visual form on the evoked response. Science, 155, 1439-1442.

Kahneman, D., Norman, J. (1964), The time-intensity relation in visual perception as a function of observer's task. Journal of Experimental Psychology, 68, 215-220.

Kahneman, D., Norman, J., \& Kubovy, M. (1967). Critical duration for the resolution of form: Centrally or peripherally determined? Journal of Experimental Psychology, 73, 323-327.

Previc, F. H., HARTER, M. R. (1982). Electrophysiological and behavioral indicants of selective attention to multifeature gratings. Perception \& Psychophysics, 32, 465-472.

Schönemann, P. H., JAmes, W. L., CArTer, F. S. (1979). Statistical inference in multidimensional scaling: A method for fitting and testing Horan's model. In J. C. Lingoes, E. E. Roskam, \& I. Borg (Eds.), Geometric representations of relational data (pp. 791-826). Ann Arbor, MI: Mathesis Press.

\section{NOTES}

1. We assumed that perceptual processing of individual dimensions (length and angle) would be complete within $\mathbf{4 0 0} \mathrm{msec}$, and we also wanted to constrain the already extensive data analysis.

2. A 50-msec increment was used to provide coverage of the complete 400 -msec interval, while still keeping the number of multidimensional scaling analyses within reasonable bounds.

3. James M. Price has pointed out to us that our error analysis procedure seems to be an application of Fisher's method of randomization, a description of which is available in Bradley (1968).

4. Three of these significantly low error scores are shown in the first three panels of Figure 1. The remaining two are for the starting points of $200 \mathrm{msec}(33 \%)$ and $250 \mathrm{msec}(33 \%)$.

(Manuscript received April 25, 1988; revision accepted for publication February 25, 1989.) 